\title{
¿Influye el tipo de maltrato sufrido en la infancia en la adopción de los mecanismos de afrontamiento adultos?
}

\section{Does the type of maltreatment suffered in childhood influence the adoption of adult coping mechanisms?}

Fecha de recepción: 26-05-2021

Fecha de aceptación: 01-12-2021

\author{
Aitana Gomis Pomares \\ Departamento de Psicología Evolutiva, Educativa, Social y Metodología. \\ Universitat Jaume I, Castellón \\ Lidón Villanueva \\ Departamento de Psicología Evolutiva y de la Educación, Social y Metodología. \\ Universitat Jaume I Castellón
}

\section{resumen/ahstract:}

Las Experiencias Adversas en la Infancia (ACEs) son un factor de riesgo para múltiples patologías tanto físicas como psicológicas, y pueden conllevar la adopción de estrategias de riesgo como el consumo de drogas, las conductas transgresoras, u otro tipo de mecanismo de afrontamiento como la creencia religiosa. Así, el objetivo de este estudio es analizar cómo influyen las ACEs en la adopción de diferentes estrategias de afrontamiento, en concreto: consumo de sustancias, fracaso escolar, creencias religiosas y alistamiento en el ejército. Los participantes, 490 jóvenes de entre 18 y 20 años, respondieron al cuestionario de Experiencias Adversas Infantiles (abuso, negligencia y disfunciones en el hogar), así como a diferentes preguntas sobre las variables de estudio. Los resultados indicaron que las estrategias de afrontamiento variaban en función del tipo de experiencia adversa sufrida. Por ejemplo, el abuso se asociaba con el consumo de drogas, la negligencia, con el fracaso escolar y las disfunciones en el hogar, con la perdida de religiosidad. En conclusión, parecen existir diferentes mecanismos de afrontamiento en función del tipo de maltrato específico, lo que puede permitir una actuación y detección mucho más temprana y eficiente por parte de los profesionales.

Adverse Childhood Experiences (ACEs) are a risk factor for multiple physical and psychological pathologies, which can lead to the adoption of risky strategies such as drug and alcohol use, deviant behaviours, or other coping mechanisms such as religious beliefs. Thus, the main objective of this study is to analyse how ACEs influence the adoption of different coping strategies, specifically: substance use, school failure, religious beliefs, and enlistment in the army. Participants were 490 young people between 18 and 20 years old, who responded to the Adverse Childhood Experiences questionnaire (abuse, neglect, and household dysfunctions), as well as to different questions about the study variables. The results indicated that coping strategies varied according to the type of adverse experience suffered. For example, abuse (physical, emotional, and sexual) was associated with drug use, neglect (physical and emotional) with school failure and dysfunction at home (alcoholic family member, domestic violence, etc.), with loss of religiosity. In conclusion, there seem to be different coping mechanisms depending on the specific type of maltreatment, which may allow for much earlier and more efficient action and detection by professionals.

\section{palabras clave/keywords:}

Experiencias adversas en la infancia, mecanismos de afrontamiento, drogas, fracaso escolar, creencias religiosas y ejército.

Adverse childhood experiences, coping mechanisms, drugs, school failure, religious beliefs, and army. 


\section{Introducción}

Las experiencias adversas en la infancia o más conocidas en inglés como Adverse Childhood Experiences (ACEs), son definidas como un conjunto de eventos negativos relacionados entre sí, junto a la falta de recursos individuales, familiares o ambientales para enfrentarlos de una forma satisfactoria, volviéndolos potencialmente traumáticos (Vega-Arce \& Nuñez-Ulloa, 2017). Dentro de estas experiencias adversas, se incluyen diferentes eventos tales como el maltrato físico o psicológico, el abuso sexual, la negligencia, ser testigo de violencia intrafamiliar, el consumo de sustancias por parte de un miembro de la familia, la separación o divorcio de los padres, la enfermedad mental o encarcelamiento de un miembro de la familia.

Las investigaciones retrospectivas realizadas en adultos indican que las ACEs son frecuentes y concurrentes (Felitti et al., 1998; Iniguez \& Stankowski, 2016). En preescolares, se estima que más del $60 \%$ ha vivido al menos una experiencia adversa (Grasso et al., 2012) y el $12.5 \%$ ha experimentado dos o más (National Center for Health, 2011). Al respecto, algunos autores establecen que la exposición a un evento adverso aumenta entre 2 y 18 veces la probabilidad de sufrir otro tipo de adversidad (Dong et al., 2004).

En este sentido, se ha observado que las personas que sufren experiencias traumáticas durante la infancia o la adolescencia tienden a presentar más problemas de salud física y mental en la edad adulta que las que no las sufren. Entre ellos pueden encontrarse problemas en el bienestar psicológico (Nurius et al., 2019), quejas somáticas, obesidad, insatisfacción sexual (Anda et al., 2006), enfermedades autoinmunes (Dube et al., 2003), e incluso la muerte prematura (Brown et al., 2009).

La conexión entre las ACEs y este enorme espectro de resultados negativos puede ser debida a la activación y el uso de mecanismos conscientes y/o inconscientes para hacer frente al estrés y la ansiedad que provoca la vivencia de experiencias adversas. En otras palabras, la adversidad en la infancia está fuertemente ligada al deterioro social, emocional y cognitivo, y a la adopción de conductas de riesgo para la salud, que promueven la aparición de estos resultados negativos (Briggs et al., 2021; Felitti, 1998; Hughes et al., 2017; Kelifa et al., 2021).

En este sentido, conductas como el consumo de alcohol y drogas o el fracaso escolar, se convierten en mecanismos de afrontamiento eficaces a corto plazo debido a sus beneficios inmediatos, así como en formas de escapar de la dramática realidad de las experiencias negativas (Dube et al., 2003). Sin embargo, a largo plazo, estas estrategias se vuelven desadaptativas y pueden conducir a resultados negativos. En esta línea, estudios anteriores sugieren que los traumas producidos en la primera infancia pueden conducir a una serie de resultados negativos para la salud como el abuso de drogas, tanto entre los adolescentes como entre los adultos (Finkelhor, 2020; Heffernan et al., 2000). Por ejemplo, se ha demostrado que el abuso físico y sexual en la infancia está asociado con el uso de drogas ilegales (Brockie et al., 2015). Además, el impacto que producen las ACEs en el consumo de drogas ilícitas, parece ser un fenómeno consistente a lo largo del tiempo (Leban \& Gibson, 2020), e incluso trasciende los cambios seculares, como la disponibilidad de drogas, las actitudes sociales hacia las drogas y las políticas preventivas (Dube et al., 2003). 
Por otro lado, también se ha observado que el maltrato infantil tiene un efecto negativo en el rendimiento académico y el éxito escolar de los niños. Cuando los investigadores comparaban a los niños maltratados con los no maltratados, descubrieron que los niños maltratados estaban menos atentos, participaban menos en clase, tenían calificaciones más bajas, presentaban peores resultados en los exámenes, suspendían más veces y tenían más probabilidades de abandonar la escuela (Leiter, 2007; Shonk \& Cicchetti, 2001). Con respecto a esto, algunos estudios han constatado que los niños que han sido desatendidos, es decir, que han sufrido negligencia física y/o emocional, presentan peores resultados académicos que los niños que han sufrido abusos físicos (Erickson \& Egeland, 1996; Jonson-Reid et al., 2004). Así pues, en una relación dosis-efecto, las ACEs estarían vinculadas a un menor rendimiento académico, al incumplimiento de los estándares del curso y a una menor puntuación en las pruebas estandarizadas de rendimiento, a dificultades de comportamiento y disciplina, y al absentismo escolar (Bethell et al., 2014).

Aparte de esto, la adversidad durante los primeros años de vida también parece interferir en el desarrollo de determinadas creencias acerca de quiénes somos y como entendemos el mundo. Así pues, algunos autores han evidenciado la interferencia que las ACEs pueden producir en el desarrollo de las creencias religiosas y espirituales entendiendo estas como aquellas ideas consideradas como verdaderas por quienes profesan una determinada religión (Dervic et al., 2006). En esta línea, la investigación sugiere que la adversidad y la religiosidad tienen una relación recíproca. Las creencias religiosas y espirituales pueden amortiguar el impacto de la adversidad, y a su vez, la adversidad experimentada durante la infancia y la adolescencia influye en las creencias religiosas en la edad adulta (Dervic et al., 2006). Por ello, los maltratos sufridos en la infancia suelen provocar la pérdida de valores religiosos y espirituales (Dervic et al., 2006; Reinert \& Edwards, 2009).

Por otro lado, el alistamiento en el ejército, también podría ser un acto instrumental para escapar de entornos familiares adversos (Blosnich et al., 2014), elevando así potencialmente la prevalencia de las ACEs entre las poblaciones militares (Sadler et al., 2004; Woodruf et al., 2006). En esta línea, estudios previos han mostrado que los hombres con un historial de servicio militar tenían más del doble de probabilidades que los hombres sin historial de servicio militar de declarar haber sido forzados a tener relaciones sexuales antes de los 18 años (Blosnich et al., 2021). En otros estudios como en del Sadler et al., (2004), se encontró que entre población militar femenina, más de la mitad reconoció haber sufrido abusos físicos o sexuales antes de alistarse en el ejército; de entre ellas, el $86 \%$ indicó que se alistó para escapar de un entorno abusivo o angustioso. De igual modo, entre mujeres veteranas militares, el 90\% habían crecido con padres perpetradores en comparación con el 10\% que se encontró en mujeres no militares (Schultz et al., 2006).

Por lo general, como se ha visto hasta el momento, es el impacto acumulativo de múltiples ACEs el que conduce a conductas de riesgo y resultados negativos en la vida adulta (Felitti \& Anda, 2010). Sin embargo, numerosos autores indican que también deben considerarse las contribuciones individuales de las ACEs específicas, ya que presentan un impacto diferencial (Agnew, 2001; Sharp et al., 2012). Por ejemplo, los niños que han sido víctimas de abuso físico tienen casi el doble de probabilidades de ser arrestados como resultado de 
haber cometido un crimen violento en la adultez (Widom \& Maxfield, 1996), o de presentar conductas transgresoras (Gomis-Pomares \& Villanueva, 2020). Por otro lado, los menores expuestos a violencia doméstica o a abuso sexual, tienen una mayor probabilidad de consumir drogas (Sharp et al., 2012), mientras que la negligencia y abuso físico se asocian positivamente con un mayor consumo de alcohol (Shin et al., 2013). Otros estudios sobre ACEs específicos incluyen que la negligencia emocional está inversamente relacionada con el rendimiento escolar (Basto-Pereira et al., 2016).

Así pues, este trabajo no solo incorpora el efecto total de las ACEs sino que, de forma adicional, se incluye el efecto diferencial de cada tipo de experiencia traumática (abuso, negligencia y disfunciones en el hogar), con la finalidad de analizar los posibles matices existentes. Asimismo, en muestra española, son escasos los estudios que han aplicado el cuestionario de experiencias infantiles adversas (ACE), de Felitti et al., (1998), por lo cual, este valor se añade a las fortalezas del estudio. Por último, conocer el papel que juegan las ACEs podría ayudarnos a comprender mejor en qué situaciones se propicia la adopción o el abandono de estrategias de afrontamiento de riesgo. De modo que, comprobar la relación entre las ACEs y las distintas estrategias adoptadas podrá ayudarnos a priorizar los recursos profesionales y económicos existentes en la prevención de las consecuencias del maltrato.

Por ello, el objetivo principal de este trabajo es explorar el poder predictivo que tiene la exposición a diferentes formas de adversidad temprana, incluyendo el abuso, la negligencia y la disfunción del hogar, en la probabilidad de adoptar diferentes mecanismos de afrontamiento como el consumo de sustancias, el fracaso escolar (número de cursos repetidos), las creencias religiosas y el alistamiento en el ejército, en el periodo de adultez emergente.

Se hipotetiza (1) una relación positiva entre la exposición a experiencias adversas en la infancia y la probabilidad de consumir drogas y alcohol, el fracaso escolar (repetir curso) y el alistamiento en el ejército en la edad adulta, (2) una relación negativa entre la exposición a experiencias adversas en la infancia y las creencias religiosas, y (3) la existencia de un impacto diferencial de los tipos de ACEs en los diferentes mecanismos de afrontamiento.

\section{Método}

\section{Participantes}

Se reclutaron 490 participantes con edades comprendidas entre los 18 y los 20 años $(M=$ 18.90 años; $D T=.77)$. Respecto a la distribución muestral, el $37.6 \%$ eran hombres y el $62.4 \%$ mujeres. La mayor parte de la muestra era de procedencia española $(92.7 \%)$ frente a un pequeño porcentaje que pertenecían a minorías étnicas de procedencia latina, rumana o africana (7.3\%). En cuanto al nivel de escolaridad, el $4.3 \%$ de los participantes no llegaron a finalizar la enseñanza secundaria, el $42.7 \%$ finalizó la enseñanza secundaria y el 53\% poseía estudios universitarios.

\section{Instrumentos}

Cuestionario Sociodemográfico. Se administró un cuestionario sociodemográfico para obtener los datos de sexo, edad, pertenencia a una minoría étnica y si se había o no repetido algún curso escolar. 
Adverse Childhood Experiences Questionnaire (ACE) (Felitti et al., 1998). Este cuestionario evalúa diferentes tipos de experiencias adversas que puede sufrir un menor durante su infancia/adolescencia. Dichas experiencias, se clasifican en tres subcategorías principales: Abuso, compuesto por abuso sexual (4 ítems), físico (4 ítems) y emocional (3 ítems); Negligencia, conformada por negligencia física (5 ítems) y emocional (3 ítems); y Disfunciones en el hogar, compuesto por vivir en un hogar con violencia doméstica (3 ítems), divorcio de los padres (1 ítems), abuso de sustancias en el hogar ( 2 ítems), enfermedad mental en el hogar ( 2 ítems) y encarcelamiento de un miembro del hogar (1 ítems). Las áreas de abuso emocional y físico, negligencia y violencia doméstica, se evaluaron según su frecuencia desde " $0=$ Nunca" a " $4=$ Con demasiada frecuencia". El resto de las experiencias adversas se evaluaron de forma dicotómica (Sí/No). Cada experiencia adversa (dimensión ACE) fue dicotomizada de acuerdo con las instrucciones del autor original (ver Felitti et al., 1998; Pinto et al., 2014). De este modo, si el sujeto puntuaba uno o más ítems de una dimensión ACE como "Frecuente" o "Muy frecuente", la categoría se consideraba presente; de lo contrario, se consideraba ausente. Estudios previos han mostrado las adecuadas propiedades psicométricas de este cuestionario (Holden et al., 2020; Murphy et al., 2014).

Ejército. Se evaluó si el participante estaba o había estado en el ejército mediante la siguiente pregunta: “Estás o alguna vez has estado en las fuerzas armadas? (ej. ejército)”. La respuesta se evaluó de forma dicotómica siendo $0=$ Sí y $1=$ No.

Religión. Mediante la pregunta "¿Tienes alguna religión?" los participantes indicaban si tenían o no alguna creencia religiosa. Esta variable también se evaluó dicotómicamente $(0=$ Sí, $1=$ No).

Consumo de drogas ilegales. Se evaluó el consumo de drogas ilegales a través de la respuesta afirmativa a la siguiente pregunta: “'Alguna vez has consumido drogas ilegales?”. Esta variable fue evaluada de forma dicotómica donde $0=$ Sí y $1=$ No.

Consumo de alcohol. Los participantes indicaban haber consumido alcohol en el último año, basándose en la siguiente pregunta: "¿Alguna vez te has emborrachado con bebidas alcohólicas?". Como en el caso anterior, la variable se midió con los valores $0=$ Sí y $1=$ No.

\section{Procedimiento}

Los datos del presente estudio forman parte del estudio Internacional SOCIALDEVIANCE1820, cuyo objetivo es el de analizar las consecuencias que las experiencias adversas infantiles tienen sobre el comportamiento pro/antisocial en el periodo de adultez emergente (para más detalles, ver Basto-Pereira et al., 2020). Los participantes fueron seleccionados mediante los métodos de muestreo por conveniencia y bola de nieve en escuelas secundarias, escuelas para adultos, universidades y organizaciones deportivas de la Comunidad Valenciana. La mayor parte de los participantes procedían de la universidad (57.14\%), seguido de ciclos formativos (28.57\%) y escuelas para adultos (14.29\%). Se seleccionaron todos aquellos sujetos con edades comprendidas entre los 18 a 20 años, independientemente de su situación laboral o de estudios y que no contaban con discapacidad intelectual, enfermedad mental o problemas con la comprensión del castellano.

Los cuestionarios eran de autoinforme, y fueron cumplimentados por los participantes mediante papel y lápiz en un periodo de tiempo de unos 30 minutos, siempre en presencia de 
los investigadores que previamente habían explicado el objetivo del estudio. Además, el Comité de Ética de la universidad correspondiente, aprobó la realización del estudio (número de referencia 22/2018). Todos los participantes dieron su consentimiento informado por escrito antes de participar en el estudio y los cuestionarios se administraron de forma colectiva, siempre en presencia de los investigadores, quienes explicaron de antemano el objetivo del estudio.

\section{Análisis de Datos}

Los análisis del presente estudio se realizaron con el programa estadístico SPSS (versión 23.0). Primeramente, se analizó la prevalencia combinada de las diferentes formas de experiencias adversas infantiles. En segundo lugar, se llevaron a cabo análisis de regresión lineal con la puntuación total de ACEs y las subcategorías ACE abuso, ACE negligencia y ACE disfunciones en el hogar sobre las variables demográficas (sexo, edad y minoría étnica), y las diferentes estrategias de afrontamiento (repetir curso, alistamiento en el ejército, creencias religiosas y el consumo de alcohol y/o drogas).

\section{Resultados}

En primer lugar, se presentan datos sobre la prevalencia española de las diferentes experiencias adversas infantiles (Tabla 1). Como se puede observar, las ACEs con mayor prevalen-

Tabla 1.- Prevalencias Combinadas ACEs

\begin{tabular}{|c|c|c|c|c|c|c|c|c|c|c|}
\hline & Dimensiones ACE & 1 & 2 & 3 & 4 & 5 & 6 & 7 & 8 & 9 \\
\hline 1. & Abuso emocional & - & & & & & & & & \\
\hline 2. & Abuso físico & $6.4 \%$ & - & & & & & & & \\
\hline 3. & Abuso sexual & $1.8 \%$ & $2.9 \%$ & - & & & & & & \\
\hline 4. & Negligencia emocional & $3.5 \%$ & $4.3 \%$ & $1.6 \%$ & - & & & & & \\
\hline 5. & Negligencia física & $2.0 \%$ & $2.7 \%$ & $1.0 \%$ & $3.3 \%$ & - & & & & \\
\hline 6. & Separación de los padres & $3.3 \%$ & $5.3 \%$ & $3.5 \%$ & $3.5 \%$ & $3.1 \%$ & - & & & \\
\hline 7. & Violencia doméstica & $2.7 \%$ & $3.1 \%$ & $1.4 \%$ & $1.9 \%$ & $1.8 \%$ & $5.1 \%$ & - & & \\
\hline $\begin{array}{l}8 . \\
\text { hog }\end{array}$ & $\begin{array}{l}\text { Abuso de sustancias } \\
\text { ar }\end{array}$ & $3.7 \%$ & $4.7 \%$ & $3.7 \%$ & $4.3 \%$ & $3.5 \%$ & $8.0 \%$ & $4.1 \%$ & - & \\
\hline 9. & Enfermedad mental familiar & $4.1 \%$ & $5.5 \%$ & $3.9 \%$ & $4.5 \%$ & $3.3 \%$ & $9.4 \%$ & $4.5 \%$ & $8.4 \%$ & - \\
\hline 10. & Encarcelamiento familiar & $1.6 \%$ & $2.0 \%$ & $1.0 \%$ & $1.6 \%$ & $1.4 \%$ & $2.0 \%$ & $2.0 \%$ & $3.3 \%$ & $2.4 \%$ \\
\hline
\end{tabular}

Nota. $\mathrm{N}=490 ; \mathrm{ACEs}=$ Adverse Childhood Experiences. 
cia forman parte de la categoría "disfunciones en el hogar", cuya combinación con mayor prevalencia era la formada por el hecho de vivir con un familiar con enfermedad mental junto con la separación o divorcio de los padres (9.4\%). Seguidamente, convivir con un familiar con enfermedad mental junto con el consumo de sustancias en el hogar (8.4\%), o el abuso de sustancias en el hogar junto con la separación o el divorcio de los padres (8.0\%) eran las siguientes combinaciones de ACEs con mayores prevalencias entre la muestra. Por último, dentro de la categoría abuso y negligencia, la combinación entre el abuso físico y el emocional era la más prevalente $(6.4 \%)$.

En segundo lugar, se realizaron análisis de regresión lineal con la puntuación total de las ACEs y las subcategorías ACE abuso, ACE negligencia y ACE disfunciones en el hogar para analizar su influencia en función de diferentes variables sociodemográficas (sexo, edad y minoría étnica), y variables como haber repetido curso, el alistamiento en el ejército, las creencias religiosas o el consumo de alcohol y/o drogas.

En este sentido, la Tabla 2 muestra que las variables que predijeron una mayor probabilidad de sufrir experiencias adversas en la infancia fueron ser mujer, haber repetido algún curso escolar o pertenecer a una minoría étnica. A su vez, el hecho de haber sufrido experiencias de maltrato en la infancia aumentaba la probabilidad de alistamiento en las fuerzas armadas, así como el consumo de drogas, mientras que disminuía la probabilidad de tener alguna religión o creencia religiosa. Este modelo explicó el 8.4\% de la varianza.

Por otro lado, teniendo en cuenta la categoría ACE abuso (formada por el abuso físico, emocional y sexual), los resultados indicaron que el hecho de ser mujer aumentaba la proba-

Tabla 2.- Análisis de Regresión Lineal de las Variables Predictoras de ACE Total

\begin{tabular}{ccccccc}
\hline & & & & \multicolumn{3}{c}{ IC 95\% } \\
& $\mathrm{B}$ & $\mathrm{SE}$ & $\mathrm{t}$ & $\mathrm{p}$ & $\mathrm{LL}$ & $\mathrm{UL}$ \\
\hline Sexo(1) & .47 & .15 & 3.08 & $.002^{*}$ & .17 & .77 \\
Edad & .14 & .10 & 1.46 & .144 & -.05 & .33 \\
Minoría étnica & .71 & .29 & 2.43 & $.015^{*}$ & .14 & 1.28 \\
Repetir curso & .21 & .11 & 1.94 & $.053^{*}$ & -.01 & .42 \\
Ejército & -4.10 & 1.11 & -3.71 & $.000^{*}$ & -6.27 & -1.93 \\
Religión & .34 & .16 & 2.15 & $.032^{*}$ & .03 & .64 \\
Drogas & -.48 & .15 & -3.11 & $.002^{*}$ & -.78 & -.18 \\
Alcohol & .30 & .22 & 1.37 & .172 & -.13 & .74 \\
\hline
\end{tabular}

Nota N=490; $\mathrm{ACE}=$ Adverse Childhood Experiences; $\operatorname{Sexo}(1)=$ Mujer; Nagelkerke $\mathrm{R}^{2}=.084$; *p<.05; SE= Standard Deviation; LL= Lower Level; UL=Upper Level. 
bilidad de haber sufrido este tipo de maltrato infantil específico. De la misma forma, haber sufrido alguna experiencia de abuso en la infancia, también aumentaba la probabilidad de alistamiento en la armada y el consumo de drogas ilegales (véase Tabla 3). Este modelo explicó el $7.9 \%$ de la varianza total.

Tabla 3. - Análisis de Regresión Lineal de las Variables Predictoras de ACE Abuso

\begin{tabular}{ccccccc}
\hline & & & & \multicolumn{3}{c}{ IC 95\% } \\
& $B$ & $S E$ & $t$ & $p$ & $L L$ & $U L$ \\
\hline Sexo(1) & .08 & .02 & 3.95 & $.000^{* *}$ & .04 & .12 \\
Edad & .02 & .01 & 1.17 & .244 & -.01 & .04 \\
Minoría étnica & .07 & .04 & 1.75 & .080 & -.01 & .15 \\
Repetir curso & .01 & .02 & .83 & .408 & -.02 & .04 \\
Ejército & -.30 & .12 & -2.46 & $.014^{* *}$ & -.55 & -.06 \\
Religión & -.02 & .02 & -.84 & .404 & -.06 & .03 \\
Drogas & -.11 & .02 & -4.99 & $.000^{* *}$ & -.15 & -.06 \\
Alcohol & .03 & .03 & 1.06 & .291 & -.03 & .09 \\
\hline
\end{tabular}

Notae:. N=490; ACE= Adverse Childhood Experiences; Sexo(1)= Mujer; Nagelkerke $\mathrm{R}^{2}=.079$; $* \mathrm{p}<.05 ; \mathrm{SE}=$ Standard Deviation; LL= Lower Level; UL=Upper Level.

Adicionalmente, cuando se analizó la categoría ACE negligencia (compuesta por la negligencia física y la emocional), los resultados obtenidos fueron los siguientes (ver Tabla 4). Primeramente, se observó una tendencia en la variable "repetir curso" que indicaba que, un mayor número de cursos repetidos se asociaba con una mayor probabilidad de haber sufrido negligencia en la infancia. Por otro lado, el hecho de haber sufrido experiencias de negligencia en la infancia aumentaba la probabilidad de alistarse en la armada o el ejército y disminuía la probabilidad de tener alguna religión o creencia religiosa. Este modelo explicó el $2.4 \%$ de la varianza total.

Finalmente, cuando se contempló la categoría ACE disfunciones en el hogar (formada por las experiencias: ser testigo de violencia doméstica, separación o divorcio de los padres, abuso de sustancias en el hogar, convivir con un familiar con enfermedad metal y encarcelamiento de un familiar), se observaron los siguientes resultados (Tabla 5). Ser mujer o pertenecer a una minoría étnica aumentaba la probabilidad de experimentar disfunciones en el hogar durante la infancia. Además, sufrir este tipo de experiencias favorecía una mayor probabilidad de ingreso en la armada o ejército y una disminución de creencias religiosas. El modelo explicó el 5.2\% de la varianza total. 
Tabla 4.- Análisis de Regresión Lineal de las Variables Predictoras de ACE Negligencia

\begin{tabular}{ccccccc}
\hline & & & & \multicolumn{2}{c}{ IC 95\% } \\
& $B$ & $S E$ & $t$ & $p$ & LL & UL \\
\hline Sexo(1) & -.01 & .02 & -.19 & .850 & .05 & .04 \\
Edad & -.01 & .01 & -.25 & .804 & -.03 & .02 \\
Minoría étnica & -.01 & .04 & -.12 & .903 & -.09 & .08 \\
Repetir curso & .03 & .02 & 1.85 & $.064 \mathrm{~T}$ & -.00 & .06 \\
Ejército & -.45 & .16 & -2.73 & $.007^{* *}$ & -.77 & -.12 \\
Religión & .07 & .02 & 2.94 & $.003^{* *}$ & .02 & .11 \\
Drogas & -.01 & .02 & -.24 & .808 & -.05 & .04 \\
Alcohol & .03 & .03 & .94 & .349 & -.03 & .09 \\
\hline
\end{tabular}

Nota.e: $\mathrm{N}=490$; $\mathrm{ACE}=$ Adverse Childhood Experiences; Sexo(1)= Mujer; Nagelkerke $\mathrm{R}^{2}=.024$; $* \mathrm{p}<.05 ; \mathrm{T}=$ Tendencia; $\mathrm{SE}=$ Standard Deviation; $\mathrm{LL}=$ Lower Level; UL=Upper Level.

Tabla 5.- Análisis de Regresión Lineal de las Variables Predictoras de ACE Disfunciones del Hogar

\begin{tabular}{ccccccc}
\hline & & & & \multicolumn{2}{c}{ IC 95\% } \\
& $\mathrm{B}$ & $\mathrm{SE}$ & $\mathrm{t}$ & $\mathrm{p}$ & $\mathrm{LL}$ & $\mathrm{UL}$ \\
\hline Sexo(1) & .04 & .02 & 2.20 & $.028^{* *}$ & .01 & .08 \\
Edad & .02 & .01 & 1.68 & .094 & -.01 & .05 \\
Minoría étnica & .01 & .04 & 2.63 & $.009^{* *}$ & .03 & .18 \\
Repetir curso & .02 & .01 & 1.52 & .130 & -.01 & .05 \\
Ejército & -.31 & .12 & -2.62 & $.009^{* *}$ & -.54 & -.08 \\
Religión & .05 & .02 & 2.34 & $.020^{* *}$ & .01 & .09 \\
Drogas & -.04 & .02 & -1.76 & .080 & -.07 & .01 \\
Alcohol & .04 & .03 & .94 & .187 & -.02 & .09 \\
\hline
\end{tabular}

Nota.e: $\mathrm{N}=490$; $\mathrm{ACE}=$ Adverse Childhood Experiences; Sexo(1)= Mujer; Nagelkerke $\mathrm{R}^{2}=.052$; $* \mathrm{p}<.05 ; \mathrm{SE}=$ Standard Deviation; LL= Lower Level; UL=Upper Level. 


\section{Discusión}

El objetivo de este trabajo era el de analizar el poder predictivo que la exposición a determinadas experiencias adversas en la infancia producía en el aumento o disminución de la probabilidad de adoptar de diferentes estrategias de afrontamiento, en concreto: el consumo de sustancias, el fracaso escolar, las creencias religiosas y el alistamiento en la armada/ejército en el periodo de adultez emergente. Los resultados obtenidos en los diferentes análisis de regresión lineal apoyaron las diferentes hipótesis postuladas.

La primera hipótesis sostenía que, a más experiencias adversas sufridas en la infancia, mayor sería la probabilidad de consumo de drogas y alcohol, de fracaso escolar y de alistamiento en el ejército. En este sentido, el hecho de haber sufrido en general, experiencias traumáticas en la infancia y en concreto abuso (físico, emocional y/o sexual), predecía el consumo de drogas en la adultez emergente. A más experiencias traumáticas o a más abuso sufrido, mayor probabilidad de consumo de drogas, tal como hallaron diversos estudios previos (Anda et al., 2006; Dube et al., 2003). Así pues, cuando hablamos de la dimensión activa (a diferencia de la negligencia, que sería la dimensión pasiva) y directa (no vicaria, como las disfunciones en el hogar) del maltrato, parece ser que los jóvenes desarrollan estrategias de afrontamiento más extremas. En consecuencia, es lógico pensar que, ante el subtipo de maltrato más intenso (el abuso físico, emocional y/o sexual) los jóvenes tratan de manejar el estrés experimentado de la forma más intensa posible con la finalidad de poder reducir ese malestar generado.

Por otro lado, el fracaso escolar entendido como el número de cursos repetidos, estaba relacionado con la negligencia como tipo de maltrato específico. De acuerdo con esto, la falta de supervisión y cuidado por parte de los progenitores podría ser un factor predictor esencial en el fracaso escolar. Los niños que han sufrido negligencia carecen de una formación adecuada y de una educación basada en el afecto que les permita desarrollar interés por el estudio, ya que no encuentran ni estímulo ni reconocimiento por sus esfuerzos. De esta manera, las carencias afectivas y la poca estimulación de los cuidadores primarios afectan al desarrollo de los niños y las niñas en el escenario educativo, tal como indica la literatura previa (Basto-Pereira et al., 2016; Bethell et al., 2014).

En cuanto al alistamiento en el ejército, se observó que tanto la puntuación de ACEs total como las diferentes dimensiones de las ACEs estudiadas en el presente trabajo (abuso, negligencia y disfunciones en el hogar) aumentaban la probabilidad de alistamiento futuro en el ejército. Así pues, como indican estudios previos, algunas personas pueden alistarse para escapar de entornos familiares violentos, abusivos o disfuncionales (Iversen et al., 2007; Woodruff et al., 2006). De este modo, en este estudio, al igual que en estudios anteriores como el de Blosnich et al., (2014), se ha podido comprobar que aquellas personas que se alistaban en el ejército eran más propensas a reportar casi todas las formas de ACEs, incluyendo disfunción doméstica y negligencia, variables menos estudiadas.

En segundo lugar, se postuló una relación inversa entre las experiencias adversas sufridas en la infancia y las creencias religiosas. Dicha hipótesis fue contrastada por los resultados, los cuales indicaron que, tanto el efecto acumulativo de ACEs como la dimensión de negli- 
gencia y de disfunciones en el hogar estaba relacionada con mayor probabilidad de ausencia de las creencias religiosas. Por consecuencia, tanto el abandono físico y/o emocional, como las disfunciones en el hogar podrían estar provocando la pérdida de esperanza y de valores religiosos y espirituales (Dervic et al., 2006; Reinert \& Edwards, 2009; Walker et al., 2009). En relación a esto, una posible explicación podría deberse al sentimiento de injusticia e incomprensión ante la pregunta: ¿por qué le pasan cosas malas a la gente buena? Frente a la imposibilidad de responder a esta pregunta muchos menores con experiencias adversas podrían perder esa fe o religiosidad, puesto que de existir un Dios o ser divino, no permitiría que este tipo de cosas les sucedieran (Walker at al., 2009).

Por último, se hipotetizó la existencia de un impacto diferencial de los diferentes ACEs específicos en la adopción de distintos mecanismos de afrontamiento. Así pues, los resultados arrojados en este estudio enfatizan dicho impacto diferencial asociando principalmente la dimensión de abuso como predictora del consumo de drogas, la dimensión de negligencia como predictora del fracaso escolar, y la dimensión de disfunciones en el hogar junto a la negligencia, como predictoras de la ausencia de creencias religiosas. Por ende, este trabajo hace hincapié en la necesidad de realizar un análisis no solo global de las experiencias traumáticas, sino diferenciado por subtipos, con la finalidad de poder captar todos los matices de la situación, puesto que, no todos los tipos de maltrato conllevan los mismos tipos de mecanismos de afrontamiento.

Por otro lado, se encontró que las variables sexo, en concreto ser mujer, y el hecho de pertenecer a una minoría étnica predecían una mayor probabilidad de sufrir experiencias adversas, en concreto, el abuso físico en el caso de las mujeres y las disfunciones en el hogar en el caso de las mujeres y las personas pertenecientes a minorías étnicas. Estos resultados coinciden con la literatura previa que indica que, las ACEs son más predominantes en mujeres que en hombres (Basto-Pereira et al., 2016; Dube et al., 2003) y en minorías étnicas (Cronholm et al., 2015).

Para finalizar, cabe mencionar ciertas limitaciones presentes en este estudio y que pueden abordarse en futuras investigaciones. Primeramente, las experiencias adversas se han evaluado a través de cuestionarios de autoinforme y con un diseño retrospectivo. Este método podría conllevar algunos errores o confusiones en los participantes a la hora de recordar experiencias traumáticas infantiles. Sin embargo, debido a que los participantes de este estudio se encuentran en el inicio del periodo de adultez emergente, son más jóvenes que en la mayoría de las investigaciones, y, por tanto, la probabilidad de que su recuerdo sea certero es mayor. Además, incluir información adicional acerca de las experiencias adversas vividas, como puede ser la frecuencia, la gravedad o la cronicidad, podría aportar información adicional con importantes implicaciones futuras. En segundo lugar, el tipo de muestreo empleado en el presente estudio (no probabilístico) constituye en sí mismo una limitación puesto que no permite que todos los individuos de la población a investigar posean las mismas oportunidades de ser seleccionados. Es decir, se escogieron solo individuos con edades entre 18 y 20 años, independientemente de su situación laboral o de estudios. Finalmente, los modelos empleados para el análisis de los datos no nos permiten establecer relaciones de causalidad sino de predicción y probabilidad. En este caso concreto, además, algunos de es- 
tos modelos predictivos presentan una baja capacidad predictiva, lo cual implica que tengan que tomarse con cautela. Como conclusión, podemos observar que existen estrategias de afrontamiento que están más relacionadas con un tipo de maltrato infantil concreto (como el consumo de sustancias con el abuso, el fracaso escolar con la negligencia y la ausencia de religiosidad con las disfunciones en el hogar), mientras que otras son más generalistas y se dan prácticamente ante cualquier tipo de maltrato (como el alistamiento en el ejército). A su vez, algunas de estas estrategias serían claramente desadaptativas e irían en detrimento de la persona como el consumo de sustancias o el fracaso escolar, pero otras como el alistamiento en el ejército o las creencias religiosas podrían resultar adaptativas, actuando como vía de canalización de la ansiedad o estrés generado por entornos familiares disfuncionales. Además, en el caso del alistamiento en el ejército, la mayoría de las personas que se alistan lo hacen por razones positivas, como el patriotismo, el altruismo y la superación personal por lo que se mostraría la vía resiliente frente a la adversidad (Woodruff, 2006).

De este modo, conociendo las estrategias adoptadas podríamos tratar de discernir qué antecedentes específicos de maltrato puede haber experimentado el sujeto y así, actuar de forma más eficaz. Esta actuación debería estar centrada sobre todo en una detección precoz por parte de los profesionales puesto que, las condiciones de salud de los adultos, como el abuso de sustancias o el fracaso escolar, pueden prevenirse si se centran los esfuerzos en la prevención primaria del maltrato en la infancia.

\section{Referencias}

Agnew, R. (2001). Building on the foundation of general strain theory: Specifying the types of strain most likely to lead to crime and delinquency. Journal of Research in Crime and Delinquency, 38(4), 319-361. https:// doi.org/10.1177/0022427801038004001

Basto-Pereira, M., Miranda, A., Ribeiro, S., \& Maia, Â. (2016). Growing up with adversity: From juvenile justice involvement to criminal persistence and psychosocial problems in young adulthood. Child Abuse \& Neglect, 62, 63-75. https://doi.org/10.1016/j.chiabu.2016.10.011

Basto-Pereira, M., Queiroz-Garcia, I., Maciel, L., Leal, I., \& Gouveia-Pereira, M. (2020). An international study of pro/antisocial behavior in young adults. Cross-Cultural Research, 54(1), 92-105. https://doi. org/10.1177/1069397119850741

Bethell, C. D., Newacheck, P., Hawes, E., \& Halfon, N. (2014). Adverse childhood experiences: assessing the impact on health and school engagement and the mitigating role of resilience. Health Affairs, 33(12), 2106-2115. https://doi.org/10.1377/hlthaff.2014.0914

Blosnich, J. R., Dichter, M. E., Cerulli, C., Batten, S. V., \& Bossarte, R. M. (2014). Disparities in adverse childhood experiences among individuals with a history of military service. JAMA Psychiatry, 71(9), 1041-1048. 1041-1048. https://doi.org/10.1001/jamapsychiatry.2014.724

Blosnich, J. R., Garfin, D. R., Maguen, S., Vogt, D., Dichter, M. E., Hoffmire, C. A., Bernhard, P. A., \& Schneiderman, A. (2021). Differences in childhood adversity, suicidal ideation, and suicide attempt among veterans and nonveterans. American Psychologist, 76(2), 284-299. https://doi.org/10.1037/amp0000755

Briggs, E. C., Amaya-Jackson, L., Putnam, K. T., \& Putnam, F. W. (2021). All adverse childhood experiences are not equal: The contribution of synergy to adverse childhood experience scores. American Psychologist, 76(2), 243-252. https://doi.org/10.1037/amp0000768 
Brockie, T. N., Dana-Sacco, G., Wallen, G. R., Wilcox, H. C., \& Campbell, J. C. (2015). The relationship of adverse childhood experiences to PTSD, depression, poly-drug use and suicide attempt in reservation-based Native American adolescents and young adults. American Journal of Community Psychology, 55(3-4), 411-421. https://doi.org/10.1007/s10464-015-9721-3

Cronholm, P. F., Forke, C. M., Wade, R., Bair-Merritt, M. H., Davis, M., Harkins-Schwarz, M., Lee, M., \& Fein, J. A. (2015). Adverse childhood experiences: Expanding the concept of adversity. American Journal of Preventive Medicine, 49(3), 354-361. https://doi.org/10.1016/j.amepre.2015.02.001

Dervic, K., Grunebaum, M. F., Burke, A. K., Mann, J. J., \& Oquendo, M. A. (2006). Protective factors against suicidal behavior in depressed adults reporting childhood abuse. The Journal of Nervous and Mental Disease, 194(12), 971-974. https://doi.org/10.1097/01.nmd.0000243764.56192.9c

Dong, M., Anda, R. F., Felitti, V. J., Dube, S. R., Williamson, D. F., Thompson, T. J., Clifton, M. \& Giles, W. H. (2004). The interrelatedness of multiple forms of childhood abuse, neglect, and household dysfunction. Child Abuse \& Neglect, 28(7), 771-784. http://dx.doi.org/10.1016/j.chiabu.2004.01.008

Dube, S. R., Felitti, V. J., Dong, M., Chapman, D. P., Giles, W. H., \& Anda, R. F. (2003). Childhood abuse, neglect, and household dysfunction and the risk of illicit drug use: the adverse childhood experiences study. Pediatrics, 177(3), 564-572. https://doi.org/10.1542/peds.111.3.564Du

Erickson, M. F., \& Egeland, B. (1996). Child neglect. In J. Briere, L. Berlinger, J. A. Bulkley, C. Jenny, \& T. Reid (Eds.), The APSAC handbook on child maltreatment (pp. 4-20). Sage.

Felitti, V. J., \& Anda, R. F. (2010). The relationship of adverse childhood experiences to adult medical disease, psychiatric disorders, and sexual behavior: Implications for healthcare. In R. Lanius \& E. Vermetten (Eds.), The impact of early life trauma on health and disease: The hidden epidemic (pp. 77-87). Cambridge University Press.

Felitti, V. J., Anda, R. F., Nordenberg, D., Williamson, D. F., Spitz, A. M., Edwards, V., \& Marks, J. S. (1998). Relationship of childhood abuse and household dysfunction to many of the leading causes of death in adults: The Adverse Childhood Experiences (ACE) Study. American Journal of Preventive Medicine, 14(4), 245-258. https://doi.org/10.1016/S0749-3797(98)00017-8

Finkelhor, D. (2020). Trends in adverse childhood experiences (ACEs) in the United States. Child Abuse \& Neglect, 108, 104641. https://doi.org/10.1016/j.chiabu.2020.104641

Gomis-Pomares, A., \& Villanueva, L. (2020). The effect of adverse childhood experiences on deviant and altruistic behavior during emerging adulthood. Psicothema, 32, 33-39. http://doi.org/10.7334/psicothema2019.142

Grasso, D. J., Ford, J. D., \& Briggs-Gowan, M. J. (2013). Early life trauma exposure and stress sensitivity in young children. Journal of Pediatric Psychology, 38(1), 94-103. http://dx.doi.org/10.1093/jpepsy/jss101

Heffernan, K., Cloitre, M., Tardiff, K., Marzuk, P. M., Portera, L., \& Leon, A. C. (2000). Childhood trauma as a correlate of lifetime opiate use in psychiatric patients. Addictive Behaviors, 25(5), 797-803. https://doi.org/10.1016/ 50306-4603(00)00066-6

Holden, G.W., Gower, T., \& Chmielewski, M. (2020). Methodological considerations in ACEs research. In Gordon GJC, Aff TO (eds) adverse childhood experiences: using evidence to advance research, practice, policy, and prevention (pp 161-182). Academic Press.

Iniguez, K. C., \& Stankowski, R. V. (2016). Adverse childhood experiences and health in adulthood in a rural population-based sample. Clinical Medicine \& Research, 14(3-4), 126-137. http://dx.doi.org/10.3127/cmr.2016.1306

Iversen, A. C., Fear, N. T., Simonoff, E., Hull, L., Horn, O., Greenberg, N., Hotopf, M., Rona, R. \& Wessely, S. (2007). Influence of childhood adversity on health among male UK military personnel. The British Journal of Psychiatry, 197(6), 506-511.

Jonson-Reid, M., Drake, B., Kim, J., Porterfield, S., \& Han, L. (2004). A prospective analysis of the relationship between reported child maltreatment and special education eligibility among poor children. Child Maltreatment, 9(4), 382-394. https://doi.org/10.1177/1077559504269192

Kelifa, M. O., Yang, Y., Carly, H., Bo, W., \& Wang, P. (2021). How adverse childhood experiences relate to subjective wellbeing in college students: The role of resilience and depression. Journal of Happiness Studies, 22(5), 2103-2123. 
Leban, L., \& Gibson, C. L. (2020). The role of gender in the relationship between adverse childhood experiences and delinquency and substance use in adolescence. Journal of Criminal Justice, 66, 101637. https://doi. org/10.1016/j.jcrimjus.2019.101637

Leiter, J. (2007). School performance trajectories after the advent of reported maltreatment. Children and Youth Services Review, 29, 363-382. https://doi.org/10.1016/j.childyouth.2006.09.002

Murphy, A., Steele, M., Dube, S. R., Bate, J., Bonuck, K., Meissner, P., Goldman, H. \& Steele, H. (2014). Adverse childhood experiences (ACEs) questionnaire and adult attachment interview (AAI): Implications for parent child relationships. Child Abuse \& Neglect, 38(2), 224-233. https://doi.org/10.1016/j.chiabu.2013.09.004

National Center for Health US (2011). Survey of Children's Health. http://www.childhealthdata.org/learn/ $\mathrm{NSCH}$.

Nurius, P. S., Fleming, C. M., \& Brindle, E. (2019). Life course pathways from adverse childhood experiences to adult physical health: A structural equation model. Journal of Aging and Health, 31(2), 211-230. https://doi. org/10.1177/0898264317726448

Pinto, R., Correia, L., \& Maia, Â. (2014). Assessing the reliability of retrospective reports of adverse childhood experiences among adolescents with documented childhood maltreatment. Journal of Family Violence, 29(4), 431-438. https://doi.org/10.1007/s10896-014-9602-9

Reinert, D. F., \& Edwards, C. E. (2009). Attachment theory, childhood mistreatment, and religiosity. Psychology of Religion and Spirituality, 7(1), 25-34. https://doi.org/10.1037/a0014894

Sadler, A. G., Booth, B. M., Mengeling, M. A., \& Doebbeling, B. N. (2004). Life span and repeated violence against women during military service: Effects on health status and outpatient utilization. Journal of Women's Health, 13(7), 799-811. https://doi.org/10.1089/jwh.2004.13.799

Schultz, J. R., Bell, K. M., Naugle, A. E., \& Polusny, M. A. (2006). Child sexual abuse and adulthood sexual assault among military veteran and civilian women. Military Medicine, 177(8), 723-728. https://doi.org/10.7205/ MILMED.171.8.723

Sharp, S. F., Peck, B. M., \& Hartsfield, J. (2012). Childhood adversity and substance use of women prisoners: A general strain theory approach. Journal of Criminal Justice, 40(3), 202-211. https://doi.org/10.1016/j.jcrimjus.2012.01.003

Shin, S. H., Miller, D. P., \& Teicher, M. H. (2013). Exposure to childhood neglect and physical abuse and developmental trajectories of heavy episodic drinking from early adolescence into young adulthood. Drug and Alcohol Dependence, 127(1-3), 31-38. https://doi.org/10.1016/j.drugalcdep.2012.06.005

Shonk, S. M., \& Cicchetti, D. (2001). Maltreatment, competency deficits, and risk for academic and behavioral maladjustment. Developmental Psychology, 37, 3-17. https://doi.org/10.1037/0012-1649.37.1.3

Walker, D. F., Reid, H. W., O'Neill, T., \& Brown, L. (2009). Changes in personal religion/spirituality during and after childhood abuse: A review and synthesis. Psychological Trauma: Theory, Research, Practice, and Policy, 1(2), 130-145. https://doi.org/10.1037/a0016211

Widom, C. S., \& Maxfield, M. G. (1996). A Prospective Examination of Risk for Violence among Abused and Neglected Children. Annals of the New York Academy of Sciences, 794(1), 224-237. https://doi. org/10.1111/j.1749-6632.1996.tb32523.x

Woodruff, T., Kelty, R., \& Segal, D. R. (2006). Propensity to serve and motivation to enlist among American combat soldiers. Armed Forces \& Society, 32(3), 353-366. https://doi.org/10.1177/0095327X05283040 